\title{
Criaderos y fauna de mosquitos en el área urbana de Armenia posterior al terremoto del 25 de enero de 1999
}

\author{
César A. Rodríguez, Diana C. Gallego, José A. González, Paola A. Luna, \\ Mitzilene Navarro, Germán A. Ramírez \\ Programa de Biología y Educación Ambiental, Universidad del Quindío, Armenia, Colombia.
}

\begin{abstract}
Como resultado del terremoto del 25 de enero de 1999 en Armenia, se creó un ambiente favorable para la proliferación de mosquitos debido al colapso de edificaciones y a la reubicación de parte de la población en áreas donde se favoreció el contacto con posibles vectores de enfermedades. Con el fin de obtener información de base para el programa de control de vectores, se llevó a cabo un muestreo entre los meses de septiembre de 1999 y febrero del 2000, para caracterizar la fauna de larvas de mosquitos en los criaderos encontrados en 14 de las 15 zonas en que fue organizada la ciudad para el proceso de reconstrucción.

Se recolectaron 7 especies de mosquitos; Aedes aegypti fue la especie más abundante en los 7 tipos de criaderos determinados en el estudio en todas las zonas muestreadas. El índice de Breteau $(10,92)$, el índice larvario $(8,22)$ y el índice de depósito $(9,44)$ determinados en este estudio, demuestran el alto riesgo de transmisión del dengue en la ciudad y, por ende, la necesidad de continuar e incrementar los esfuerzos de control del vector de la enfermedad.
\end{abstract}

Palabras clave: dengue, criaderos, mosquitos, Aedes aegypti, Armenia.

Mosquitoe breeding sites and fauna in Armenia's urban area after the January 25, 1999, earthquake

As a result of the January 25, 1999, earthquake in the city of Armenia, a favorable environment for mosquito breeding was created due to the collapse of buildings and the relocation of some of the people in areas where contact with possible vectors of disease was more favorable. To obtain baseline information for a vector control program, a survey was conducted to characterize the mosquito larvae fauna in water holding containers in 14 of the 15 zones in which the city was organized for the reconstruction process.

Seven mosquito species were collected, Aedes aegypti being the most abundant one in the seven kinds of containers examined and in all of the zones studied. The Breteau index (10.92), the larval index (8.22), and the deposit index (9.44) determined in this study demonstrated the high risk for dengue transmission in the city and the need to continue and increase the efforts to control the mosquito vector.

Key words: dengue, container, mosquito, Aedes aegypti, Armenia.

La presencia de dípteros de la familia Culicidae en un ambiente determinado, indica áreas de riesgo potencial en materia de salud pública, ya que varias de sus especies son vectoras de enfermedades (1). De estas especies, Aedes aegypti es la especie más importante por ser un mosquito de hábitos domésticos que se cría en

Correspondencia:

César Rodríguez ceroto@uniquindio.edu.co

Recibido: 26/12/00; aceptado: 06/04/01 depósitos de agua artificiales y, secundariamente, en depósitos naturales en áreas urbanas (2), y a que es responsable de la transmisión del dengue en su forma clásica y hemorrágica, además de la fiebre amarilla.

Compartiendo el hábitat con A. aegypti, se pueden encontrar otras especies de mosquitos, algunos de los cuales se consideran transmisores de diversas patologías a los vertebrados, incluido el hombre (3), y otras consideradas de importancia como agentes de control biológico. 
Durante 1998, en el municipio de Armenia se presentaron 2.853 casos de dengue, de los cuales 1.737 fueron clásicos y 537 hemorrágicos, lo que llevó a la clasificación del dengue en el departamento de Quindío como una epidemia. Para 1999, disminuyó la incidencia de la enfermedad, gracias a la labor del programa de control de vectores de la Secretaría Municipal de Salud; se presentaron 1.068 casos de dengue clásico y 31 de dengue hemorrágico (4).

Después del sismo del 25 de enero de 1999, la proliferación de sitios aptos para la cría de vectores por efecto del colapso de las edificaciones y el incremento del contacto entre personas y vectores, así como los casos de dengue registrados en el municipio de Armenia, determinaron la necesidad de evaluar la densidad de los vectores y la presencia de criaderos en el área urbana.

\section{Materiales y métodos}

\section{Sitio de muestreo}

El municipio de Armenia, capital del departamento de Quindío, está localizado en la zona andina a los $4^{\circ} 32^{\prime}$ latitud norte y $75^{\circ} 4^{\prime}$ longitud oeste (figura 1), a una altura de $1.483 \mathrm{msnm}$, con una temperatura promedio de $20^{\circ} \mathrm{C}$ y una precipitación anual promedio de $2.500 \mathrm{~mm}$. El área urbana tiene 2.253 hectáreas y una población de 303.098 habitantes (5).

Después del sismo del 25 de enero de 1999, la ciudad fue zonificada por el Fondo de Reconstrucción para el Eje Cafetero (FOREC) (figura 1) en 15 áreas o gerencias zonales manejadas por organizaciones no gubernamentales (ONG), las cuales se encargarían de la reconstrucción en cada zona. La zonificación está basada en los lineamientos del Plan de Ordenamiento Territorial para 1999. Estas zonas, excepto la zona 15 (considerada como no potencial para la formación de criaderos), fueron muestreadas durante los meses de septiembre, octubre y noviembre de 1999 (período de lluvias) y en los meses de enero y febrero del 2000 (período seco).

De acuerdo con la metodología para el reconocimiento geográfico y muestreo contenida en el plan de contingencia contra el dengue (6), se decidió hacer muestreos en la zona urbana de

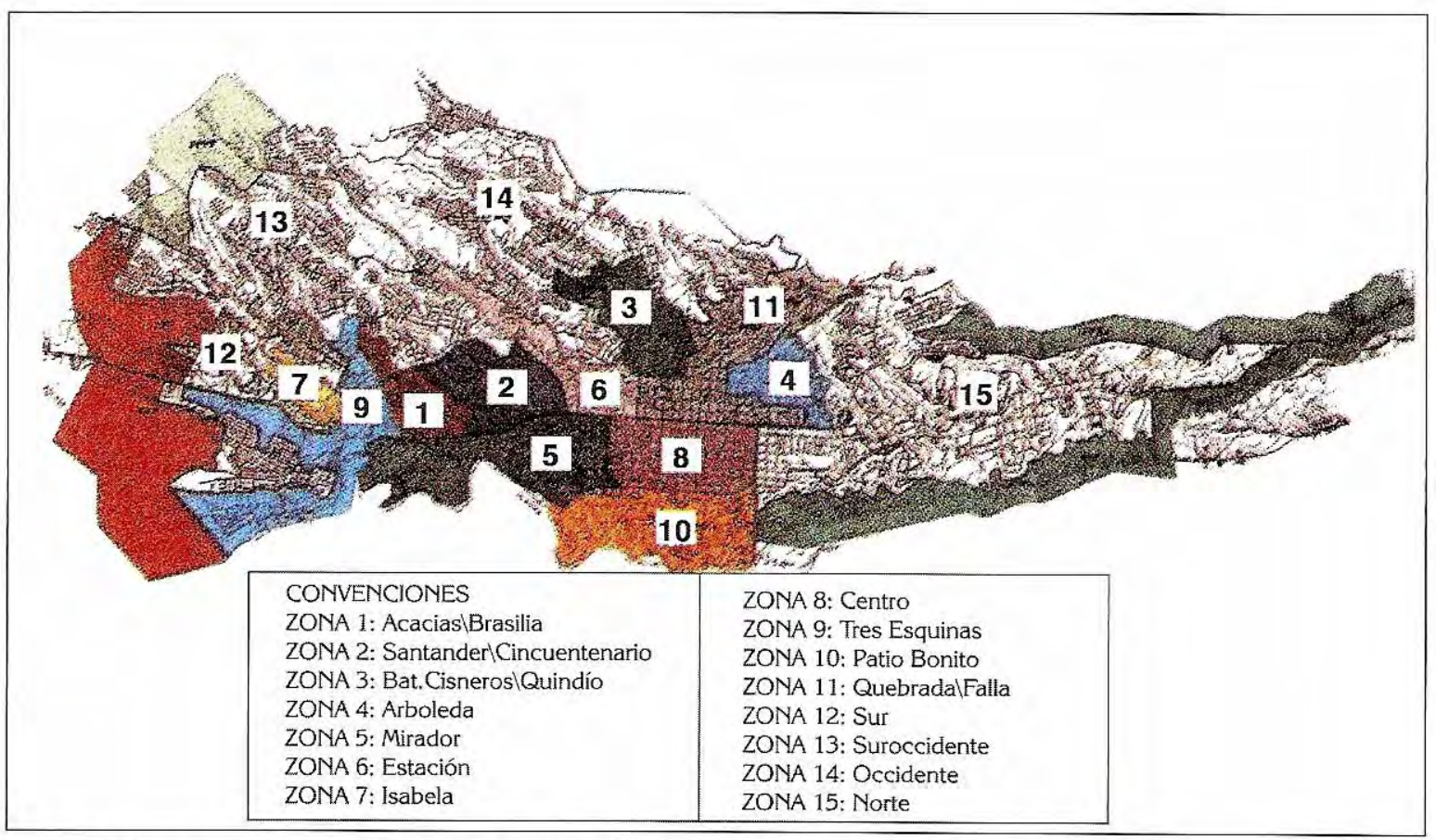

Figura 1. Mapa del municipio de Armenia con las 16 gerencias zonales establecidas por el FOREC. 
Armenia con intervalos de cada tres casas. Se evaluaron 1.932 casas, tamaño de muestra superior al recomendado (7), ya que para una localidad del tamaño de Armenia se recomiendan 1.000 casas, aproximadamente.

\section{Método de recolección de especímenes}

La búsqueda de las formas inmaduras de tercer y cuarto estadio se llevó a cabo inspeccionando todos los criaderos potenciales tanto artificiales como naturales. Los datos obtenidos se consignaron en el formulario nacional No. 1 para el registro de la encuesta larvaria. Se utilizó el método del INS (8) para la recolección de las formas inmaduras. Se sumergió un cucharón tres veces en el criadero y se recolectaron alrededor de 5 larvas, utilizando un gotero. Cuando el recipiente era pequeño o contenía poca agua, las larvas se recolectaron directamente con el gotero. Las larvas recolectadas se guardaron en viales de $5 \mathrm{ml}$ con alcohol al $70 \%$ para la posterior identificación en el laboratorio.

\section{Determinación taxonómica}

Para la determinación de las larvas, se emplearon las claves de Lane (9) y Tinker y Olano (10).

\section{Análisis de datos}

Los datos de prevalencia de larvas en los criaderos se sometieron a análisis de varianza bajo un diseño de bloques al azar, para establecer si la prevalencia de larvas era igual en los diferentes criaderos. Para el efecto, se consideraron las zonas evaluadas como bloques, pues se supone que la prevalencia es diferente entre una zona y otra. Como los datos están en porcentajes y hay valores cero, se realizó la transformación $\sqrt{ } X+0,5$ (7). Con el fin de establecer cuál o cuáles depósitos diferían entre sí, se realizó la prueba de DVS de Tukey con un $\alpha=5 \%$ (11).

Para la evaluación de la infestación con A. aegypti, se utilizaron los siguientes índices:

Indice larvario $(\mathrm{IL})=$ (casas positivas/casas inspeccionadas) $\times 100$.

Indice de depósito (ID)=(depósitos positivos/ depósitos inspeccionados) x 100 .

Indice de Breteau=(depósitos positivos x 100)/ casas inspeccionadas.
El índice larvario es el más utilizado para medir los niveles de población, pero no considera el número de recipientes positivos ni su productividad y el índice de depósito sólo proporciona información sobre la proporción de recipientes con agua que resultan positivos. El índice de Breteau establece una relación entre los recipientes positivos y las viviendas y se le considera el más informativo (7).

\section{Resultados}

Como resultado del colapso o la destrucción parcial de las construcciones a raíz del sismo del 25 de enero, se generaron zonas de escombros que dieron lugar a charcas temporales de agua en las grietas de los pisos, en las segundas plantas de inmuebles desprovistos de techo y en los escombros expuestos a la intemperie, entre otros. Después del sismo se incrementó el uso de recipientes para almacenar agua en los asentamientos temporales y el empleo de guadua como material de construcción. Por tanto, se detectaron tres nuevos tipos de depósitos de agua: escombros, guadua y recipientes, adicionales a los cuatro tipos de criaderos (tanque bajo, llanta, planta y miscelánea) considerados en los muestreos de rutina de la Secretaría de Salud Municipal. El cuadro 1 muestra las especies de culícidos encontradas en los diferentes tipos de criaderos.

Se determinó un total de 7 especies de culícidos en el área urbana del municipio de Armenia: $A$. aegypti, Aedes fluviatilis, Limatus durhami, Culex nigripalpus, Culex quinquefasciatus, mosquitos del complejo Culex coronator y una especie no identificada del género Toxorhynchites. La especie con el mayor número de larvas recolectadas fue $A$. aegypticon 425 individuos, encontrados en las 14 zonas, seguida de la especie $C$. quinquefasciatus con 78 individuos, encontrados en 11 zonas de la ciudad (figura 2, cuadro 2). El género Toxorhynchites presentó el menor número con 3 individuos, recolectados en la zona 10 y en criaderos de guadua.

La especie $A$. aegypti se encontró desarrollando sus estados larvarios en todos los tipos de criaderos o depósitos determinados para este estudio (tanque bajo, planta, llanta, escombros, guadua, recipientes y miscelánea) y compartiendo 
Cuadro 1. Especies de larvas de mosquito recolectadas en el área urbana del municipio de Armenia (septiembre/99febrero/00).

\begin{tabular}{|c|c|c|c|c|c|c|c|}
\hline \multirow[t]{2}{*}{ Especie } & \multicolumn{7}{|c|}{ Tipo de depósito } \\
\hline & $\begin{array}{c}\text { Tanque } \\
\text { bajo }\end{array}$ & Plantas & Llanta & Escombros & Guadua & Recipientes & Miscelánea \\
\hline Aedes aegypti & + & + & + & + & + & + & + \\
\hline Aedes fluviatilis & + & & & + & + & + & \\
\hline Limatus durhami & + & & + & + & & & + \\
\hline Culex nigripalpus & & & & + & & + & + \\
\hline Complejo Culex coronator & + & & & + & & + & \\
\hline Culex quinquefasciatus & + & & + & + & & & \\
\hline Toxorhynchites & & & & & + & & \\
\hline
\end{tabular}

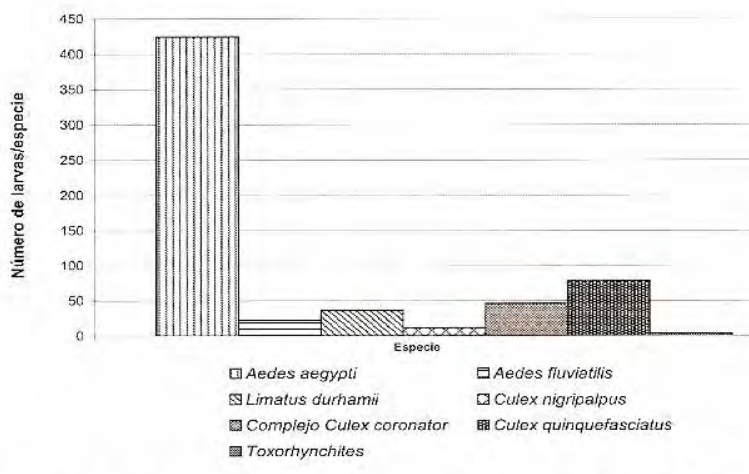

Figura 2. Número de individuos por especie de culícidos en el área urbana del municipio de Armenia (septiembre/99febrero/00).

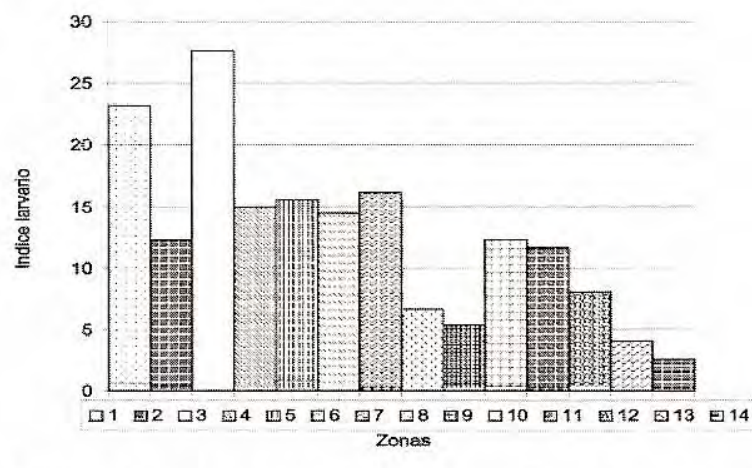

Figura 3. Indice de infestación con larvas de $A$. aegypti en criaderos de 14 zonas del municipio de Armenia (septiembre/99-febrero/00).

Cuadro 2. Distribución porcentual de depósitos según prevalencia de larvas en 14 zonas del municipio de Armenia (septiembre/99-febrero/00).

\begin{tabular}{llc}
\hline Especie & \multicolumn{1}{c}{ Zonas } & $\begin{array}{c}\text { Porcentaje de zonas } \\
\text { por especie }\end{array}$ \\
\hline Aedes aegypti & $1,2,3,4,5,6,7,8,9,10,11,12,13,14$ & 100 \\
Aedes fluviatilis & $1,2,5,6,10,11$ & 42,85 \\
Limatus durhami & $3,4,5$ & 21,42 \\
Culex nigripalpus & $1,3,11,12$ & 21,42 \\
Complejo Culex Coronator & $1,2,3,4,6,7,8,9,10,13,14$ & 78,57 \\
Culex quinquefasciatus & $1,2,3,5,6,7,9,11,12,13,14$ & 78,57 \\
Toxorhynchites & 10 & 7,14 \\
\hline
\end{tabular}

las zonas examinadas con las demás especies determinadas (cuadro 1).

De un total de 1.932 casas inspeccionadas, 159 fueron positivas para infestación larvaria y se obtuvo un índice larvario de 8,22. Se inspeccionaron 2.233 depósitos con agua, de los cuales 211 fueron positivos, para un índice de depósito de 9,44. El índice de Breteau o número de depósitos positivos por cada 100 casas fue de
10,92. Los índices fueron calculados también para cada una de las zonas, obteniéndose el mayor índice larvario en las zonas 3 y 1 con 27,6 y $23,2 \%$ de casas con $A$. aegypti, respectivamente; las zonas 13 y 14 presentaron el menor índice larvario con el 3,97 y el 2,54\% (figura 3).

La zona con mayor índice de depósito fue la 1 con $52,6 \%$ de depósitos positivos, seguida de la zona 3 con $23,7 \%$. Las zonas 13 y 14 registraron 
el menor índice de depósito con el 3,82 y 2,5\%, respectivamente (figura 4 ). El índice de Breteau más alto se presentó en la zona 1 con $89,2 \%$ y el más bajo en la zona 14 con $2,79 \%$ de depósitos positivos por cada 1.000 casas (figura 5).

La prueba de DVS de Tukey mostró diferencias significativas entre los promedios de prevalencia de los criaderos recipientes y tanque bajo. Los promedios de prevalencia de larvas en escombros,

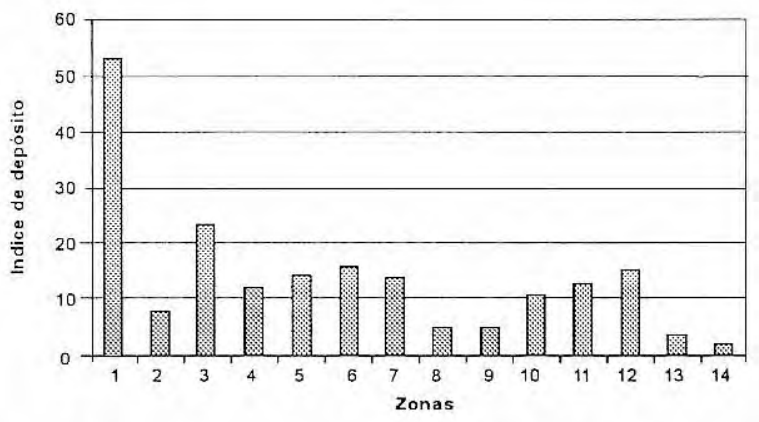

Figura 4. Indice de depósito en 14 zonas del municipio de Armenia (septiembre/99-febrero/00).

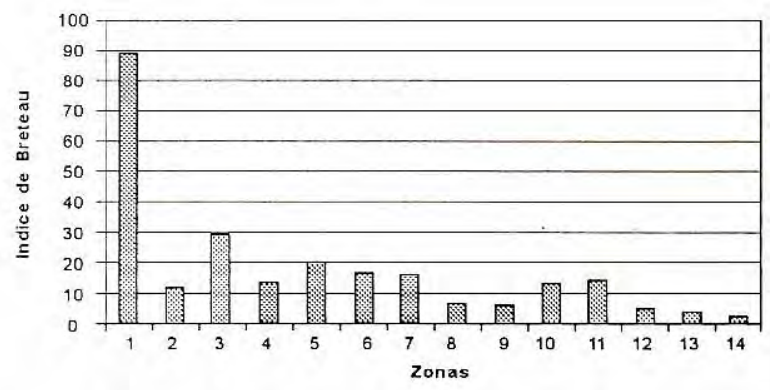

Figura 5. Indice de Breteau en 14 zonas del municipio de Armenia (septiembre/99-febrero/00).

Cuadro 3. Prueba de DVS de Tukey, comparación de medias de prevalencia de larvas según el criadero.

\begin{tabular}{lcc}
\hline Criaderos & $\begin{array}{c}\text { Promedio } \\
\text { (larvas por criadero) }\end{array}$ & Significancia* \\
\hline Tanque bajo & 2,84 & $*$ \\
Escombro & 4,520 & Ns \\
Miscelánea & 4,729 & $\mathrm{Ns}$ \\
Llanta & 5,064 & $\mathrm{Ns}$ \\
Guadua & 5,855 & $\mathrm{Ns}$ \\
Recipiente & 7,170 & $*$ \\
\hline
\end{tabular}

${ }^{*} \mathrm{P} \leq 0,05$, significativo

NS=no significativo guadua, Ilanta, recipiente y miscelánea no difieren significativamente entre sí (cuadro 3 ).

\section{Discusión}

Las especies encontradas asociadas con A. aegypti en este estudio son las mismas encontradas en el municipio de Guaduas, Cundinamarca, por Olano y Tinker en 1993 (3). Según estos autores, la especie Aedes fluviatus está asociada con $A$. aegypti en criaderos artificiales en Brasil y se ha aislado de esta especie el virus responsable de la fiebre amarilla. La especie Limatus durhami se ha informado en ambientes domésticos en Panamá y Brasil (3). La especie C. quinquefasciatus se considera vector de filariasis y algunas encefalitis en otras latitudes y, en Brasil, ha sido aislado de esta especie el virus Oropouche, causante de enfermedad febril en humanos (2). Las especies Culex coronator y Culex nigripalpus se han encontrado en criaderos urbanos en Cali (12) y en criaderos rurales en La Guajira, donde también se encontraron adultos de Toxorhynchites (13).

Las larvas de mosquitos del género Toxorhynchites se encuentran generalmente en huecos en los árboles (en guadua en nuestro estudio) y son predadoras de otras larvas de mosquitos.

En estudios llevados a cabo en diferentes regiones del país, se registra el hallazgo de otras especies vectoras como Aedes albopictus, vector del dengue, en Leticia, Amazonas (14); Aedes taeniorhynchus y Psorophora confinnis, vectores del virus de la encefalitis equina, encontrados en Tolima (15) y La Guajira (16), respectivamente, y Haemagogus equinus, vector del virus de la fiebre amarilla selvática, encontrado en zonas urbanas de Bucaramanga (17), Fonseca (La Guajira), Guaduas (Cundinamarca) y Ortega (Tolima), donde se le ha encontrado compartiendo hábitat con $A$. aegypti $(18,19)$. Ninguna de estas especies de mosquitos se encontró en el área urbana de Armenia, pero su presencia en otras partes del país sugiere la posibilidad de dispersión a otras zonas y la necesidad de continuar con los procesos de vigilancia de otros posibles vectores.

Debe anotarse, además, que en la zona rural de Armenia se identificaron 21 criaderos positivos para Anopheles spp. en 1999, resultado de la 
búsqueda iniciada luego de presentarse 11 casos de malaria en el municipio (4).

Los resultados del análisis de varianza y la comparación de medias de prevalencia de larvas según el depósito determinaron que los recipientes de almacenamiento de agua son el tipo de criadero con mayor prevalencia de larvas. Esta situación se debió, posiblemente, al hecho de que en el período de muestreo el almacenamiento fue la alternativa para el abastecimiento de agua utilizada por gran parte de la población, retardando los períodos de recambio y lavado de los depósitos de agua y favoreciendo, así, la oviposición y el desarrollo de las larvas. Se comprobó, además, que los escombros de casas abandonadas fueron criaderos óptimos, principalmente para la especie A. aegypti.

Los valores altos de los índices de infestación y el hecho de que la especie con mayor distribución en las 14 zonas inspeccionadas fue $A$. aegypti, indican que el riesgo de brotes epidémicos de dengue es alto y que la tendencia probablemente sea hacia el aumento de los índices debido a que el control se hace más difícil en los dos tipos de criaderos que presentaron mayor prevalencia de larvas.

El riesgo se confirma con los datos obtenidos en el 2000 para Armenia, donde se informa un total de 959 casos de dengue, de los cuales $17(1,8 \%)$ fueron considerados dengue hemorrágico, pero sin verificación de laboratorio (20). Estos datos son similares a los de 1999, lo cual indica que, a pesar de los esfuerzos de control, no se ha logrado una reducción significativa del vector del dengue, debido, posiblemente, a las nuevas circunstancias creadas por el sismo.

Los índices larvarios son una indicación deficiente de la producción de adultos (7); por tanto, se recomienda el empleo de otros procedimientos alternos como la monitorización de adultos, que proporciona otros datos como las tendencias estacionales de la población, la dinámica de la transmisión o la evaluación de las intervenciones para el control del mosquito adulto. Otro método sensible y más económico para detectar la presencia de $A$. aegypti son las trampas de ovipostura, especialmente útiles para la detección precoz de nuevas infestaciones en zonas de las cuales se ha eliminado el mosquito (7).

Para controlar el problema de vectores en Armenia, se requiere continuar con las medidas de control integrado y con las campañas educativas de prevención, así como con la vigilancia entomológica, manteniendo actualizada la información sobre las especies de mosquitos vectores e implementar otros procedimientos para detectar oportunamente la introducción de otras especies, adelantar las acciones adecuadas de control vectorial y evaluar los resultados.

\section{Agradecimientos}

Se agradece el apoyo logístico recibido por el Grupo de Vectores y Zoonosis de la Secretaría de Salud Municipal de Armenia; a Jorge Enrique García por la asesoría estadística y al personal del Laboratorio de Biología de la Universidad del Quindío por la colaboración.

\section{Referencias}

1. Villarreal L, González .. Culicidae (Diptera) larvas de especies de vectores en Colombia. En: Rincón ME, Pardo $\mathrm{RH}$, Ospina R, Muñoz P, editores. Seminario de invertebrados acuáticos y su utilización en estudios ambientales. Bogotá, D.C.: SOCOLEN; 1997. p.49-73.

2. Kettle D. Medical and veterinary entomology. Second edition. Wallington: CAB International; 1995.

3. Olano VA, Tinker ME. Fauna de mosquitos asociada con $A$. aegypti en Guaduas, Colombia, S.A. Biomédica 1993;13:71-4.

4. Secretaría Municipal de Salud Pública. Informe epidemiológico 1999. Armenia, Quindío: Secretaría Municipal de Salud Pública; 1999.

5. Departamento Administrativo Nacional de Estadística. Censo Nacional de 1999. Bogotá, D.C.: Dane; 1999.

6. Instituto Nacional de Salud, Organización Panamericana de la Salud. Vigilancia y control del $A$. aegypti en el plan de contingencia contra el dengue. Bogotá, D.C.: Ministerio de Salud; 1998.

7. Organización Panamericana de la Salud. Dengue y dengue hemorrágico en las Américas: guías para su prevención y control. Washington, D.C.: OPS; 1995.

8. Subdirección de Ambiente y Salud. Vigilancia entomológica del Aedes aegypti. Bogota, D.C.: Ministerio de Salud; 1998.

9. Lane J. Neotropical Culicidae. Sao Paulo: Editora da Universidade de Sao Paulo; 1953. 
10. Tinker ME, Olano VA. Clave práctica para las larvas de mosquitos neotropicales en recipientes (mimeógrafo). Bogotá, D.C.: Instituto Nacional de Salud; 1995.

11. Steel R, Torrie J. Bioestadística. Segunda edición. México: McGraw-Hill; 1998.

12. Fajardo P, Arias A. Mosquitos comunes en Cali (Colombia). Colombia Médica 1981;12:20.

13. Morales A, Ferro C, Isaza de Rodríguez C, Cura E. Encuesta sobre artrópodos de interés médico en La Guajira, Colombia, Suramérica. Biomédica 1987;7:8794.

14. Vélez ID, Quiñones ML, Suárez M, Olano V, Murcia LM, Correa E, et al. Presencia de Aedes albopictus en Leticia, Amazonas, Colombia. Biomédica 1998;18:192-8.

15. Olano VA. Hallazgo de Aedes taeniorhynchus (Wiedemann, 1921) en un lugar del municipio de Ambalema, departamento del Tolima (Colombia) (Diptera: Culicidae). Biomédica 1985;5:26-8.
16. Morales A, Romero M, Olano VA, de Calvache D. Demostración del virus de la encefalitis equina venezolana tipo enzoótico en la hemolinfa de mosquitos Psorophora confinnis infectados por vía oral. Biomédica 1982;3:111-7.

17. Olano VA, Bueno A, González CJ, Correa MC. Hallazgo del mosquito Haemagogus equinus (Theobald, 1903) en el área metropolitana de Bucaramanga, Santander. Biomédica 1997;17:128-31.

18. Morales A. Distribución geográfica, horizontal y vertical de Haemagogus (Diptera: Culicidae) de Colombia. Rev Acad Cien Fis Exac y Nat 1968;13:50:267-72.

19. Morales A, Ferro MC, Isaza C, Cura E. Búsqueda de mosquitos del género Haemagogus en el departamento de La Guajira, Colombia, S.A. (Diptera: Culicidae). Biomédica 1984;4:25-6.

20. Secretaría Municipal de Salud Pública. Informe epidemiológico 2000. Armenia, Quindío: Secretaría Municipal de Salud Pública; 2000. 